$\mathrm{IC} / 95 / 159$

July 1995

\title{
ON SYMMETRY NON-RESTORATION AT HIGH TEMPERATURE
}

\author{
G. Bimonte \\ and \\ G. Lozano \\ International Centre for Theoretical Physics, P.O.BOX 586 \\ I-34100 Trieste, ITALY
}

\begin{abstract}
We study the effect of next-to-leading order contributions on the phenomenon of symmetry non-restoration at high temperature in an $O\left(N_{1}\right) \times O\left(N_{2}\right)$ model.
\end{abstract}

\footnotetext{
${ }^{1}$ E-mail addresses: Bimonte@ictp.trieste.it , Lozano@ictp.trieste.it
} 
It is a well known fact that broken symmetries are usually restored at sufficiently high temperatures [1, 2, 3]. Nevertheless, as already noticed by Weinberg [2], there are models, like the $O(N) \times O(N)$ scalar theory, for which the high temperature phase is less symmetric than the low temperature one in a certain region of the parameter space.

As noticed by Mohapatra and Senjanovic 㕶, the effects of symmetry non restoration can have interesting phenomenological implications in connection to the CP problem. The same phenomenon has also been used to provide non inflationary solutions to the monopole problem, as argued by Langacker and Pi [5] and more recently by Dvali et al. [6].

Weinberg's analysis of the problem [2] is based on a lowest order perturbative calculation of the thermal masses, the region of parameters giving symmetry non restoration being associated to the values of the coupling constants giving imaginary values for some thermal masses.

In this letter, we will compute the next-to-leading order contributions to the thermal masses and analyze how the region of symmetry non-restoration gets modified by their inclusion. The reason for doing this is twofold: from one side, as these models contain more than one coupling constants, it may very well happen that next-to-leading order effects dominate over the leading ones, in certain regions of the parameter space. On the other side, when trying to achieve symmetry-non restoration in realistic models, such as those considered in ref.[6], one may have to take values of the coupling constants large enough for next-to-leading order corrections to become significant.

For simplicity, we will discuss here only the simplest model presenting this phenomenon, that is, when the $O\left(N_{1}\right) \times O\left(N_{2}\right)$ symmetry is global. In this case, the theory is described by the renormalized euclidean Lagrangian

$$
L_{R}=\frac{1}{2} \partial_{\mu} \phi_{1} \partial_{\mu} \phi_{1}+\frac{1}{2} \partial_{\mu} \phi_{2} \partial_{\mu} \phi_{2}+V\left(\phi_{1}, \phi_{2}\right)+L_{c t}
$$

where $\phi_{1}\left(\phi_{2}\right)$ is a $N_{1}\left(N_{2}\right)$ components real vector, $V\left(\phi_{1}, \phi_{2}\right)$ is a $O\left(N_{1}\right) \times O\left(N_{2}\right)$ symmetric potential:

$$
V\left(\phi_{1}, \phi_{2}\right)=\frac{1}{2} m_{1}^{2} \phi_{1} \phi_{1}+\frac{1}{2} m_{2}^{2} \phi_{2} \phi_{2}+\frac{1}{4 !} \lambda_{1} \mu^{2 \epsilon}\left(\phi_{1} \phi_{1}\right)^{2}+\frac{1}{4 !} \lambda_{2} \mu^{2 \epsilon}\left(\phi_{2} \phi_{2}\right)^{2}+\frac{1}{4} \lambda \mu^{2 \epsilon}\left(\phi_{1} \phi_{1}\right)\left(\phi_{2} \phi_{2}\right)
$$

and $L_{c t}$ stands for the counterterms Lagrangian, calculated in the $\overline{M S}$ scheme ( $\mu$ is the dimensional 
regularization scale parameter and $\epsilon=\frac{4-d}{2}$ ). As we shall see later, the crucial fact for symmetry nonrestoration is that the mixing coupling constant $\lambda$ can take negative values. In order for the potential to be bounded from below, the coupling constants have to satisfy the following relation

$$
\lambda_{1} \lambda_{2}>9 \lambda^{2}
$$

We will show how next-to leading order corrections to the thermal masses are calculated, by first looking at the simpler $O(N)$ case, which can be obtained from the above Lagrangian by simply setting $\phi_{2}=0$.

$$
L_{R}=\frac{1}{2} \partial_{\mu} \phi_{1} \partial_{\mu} \phi_{1}+\frac{1}{2} m_{1}^{2} \phi_{1} \phi_{1}+\frac{1}{4 !} \lambda_{1} \mu^{2 \epsilon}\left(\phi_{1} \phi_{1}\right)^{2}+L_{c t} .
$$

As explained in [2], perturbation theory based on this Lagrangian breaks down at high temperatures as powers of the temperature can compensate for powers of the coupling constants making radiative corrections large. The remedy to this problem consists in a redefinition of the mass and the introduction of a compensating counterterm

$$
L_{R}=\frac{1}{2} \partial_{\mu} \phi_{1} \partial_{\mu} \phi_{1}+\frac{1}{2} M^{2} \phi_{1}^{2}+\frac{1}{4 !} \lambda_{1} \mu^{2 \epsilon}\left(\phi_{1} \phi_{1}\right)^{2}+L_{c t}-\frac{1}{2} \sigma^{2} \phi_{1} \phi_{1}
$$

where

$$
M^{2}=m^{2}+\sigma^{2}
$$

and $\sigma^{2}$ is a temperature dependent counterterm (not to be confused with the mass renormalization counterterm already contained in $L_{c t}$ ) which should be determined self consistently in such a way to eliminate from the self-energy the terms which diverge quadratically with the temperature. To illustrate how the mechanism works, it is sufficient to consider the case $m^{2} \geq 0$. To one loop, one has for the self energy at zero momentum,

$$
\Sigma=-\lambda_{1} \frac{2+N_{1}}{96 \pi^{2}} M^{2} \ln \left(\frac{M^{2}}{4 \pi \mu^{2}}\right)-\lambda_{1} \frac{2+N_{1}}{3} T^{2} h\left(\frac{M}{T}\right)+\sigma^{2}
$$

Here $h$ is defined as:

$$
h(y)=\frac{1}{4 \pi^{2}} \int_{0}^{\infty} d x \frac{x^{2}}{\left(x^{2}+y^{2}\right)^{\frac{1}{2}}\left(e^{\left(x^{2}+y^{2}\right)^{\frac{1}{2}}}-1\right)} .
$$


For small values of $y, h(y)$ has the asymptotic expansion

$$
h(y)=\frac{1}{24}-\frac{1}{8 \pi} y-\frac{1}{16 \pi^{2}} y^{2}\left(\ln \frac{y}{4 \pi}+\gamma-\frac{1}{2}\right),
$$

$\gamma$ being the Euler constant. Notice that in eq.(7) we have subtracted the following UV divergent part which is cancelled by the mass counterterm in the $\overline{M S}$ scheme:

$$
\Sigma^{U V}=-\lambda_{1} M^{2} \frac{2 N_{1}+1}{96 \pi^{2}}\left[\frac{1}{\epsilon}+1-\gamma\right] .
$$

From eq.(17), one derives the "gap equation":

$$
\frac{M^{2}}{T^{2}}=\frac{m^{2}}{T^{2}}+\lambda_{1} \frac{\left(2+N_{1}\right)}{3} h\left(\frac{M}{T}\right),
$$

which, after making use of eq.(9), becomes

$$
\frac{M^{2}}{T^{2}}=\frac{m^{2}}{T^{2}}+\lambda_{1} \frac{\left(2+N_{1}\right)}{3}\left(\frac{1}{24}-\frac{M}{8 \pi T}\right)+O\left(\lambda_{1} \frac{M^{2}}{T^{2}} \ln \frac{M}{T}\right) .
$$

Solving this equation to lowest order gives the well known thermal mass,

$$
M^{2}=m^{2}+\frac{2+N_{1}}{3} \frac{1}{24} \lambda_{1} T^{2}
$$

This choice of $M$ would in principle restore the validity of perturbation theory for $\frac{T}{m} \gg 1$ in the sense that higher loops corrections will be suppressed by powers of $\lambda$ with respect to the "tree level" thermal mass eq.(13). Notice that when eq.(13) is introduced in eq.(7), one gets at high temperatures $(T \gg m)$

$$
\Sigma=\frac{3}{\pi}\left(\frac{2+N_{1}}{3} \frac{\lambda_{1}}{24}\right)^{\frac{3}{2}} T^{2}
$$

This term is the next to leading order correction to the self-energy, and although it is not immediately obvious, does not get modified by higher loops corrections, as they are at least of order $\lambda_{1}^{2} \ln \lambda_{1}$ (see ref. [7] for details).

Notice that the same contribution could have been obtained more directly by just solving the gap equation to next to leading order, that is, by keeping the term linear in $\frac{M}{T}$,

$$
\frac{M^{2}}{T^{2}}=\frac{m^{2}}{T^{2}}+\lambda_{1} \frac{\left(2+N_{1}\right)}{3}\left(\frac{1}{24}-\frac{M}{8 \pi T}\right)
$$


giving, for $\frac{T}{M} \gg 1$

$$
M^{2}=\frac{2+N_{1}}{3} \frac{\lambda_{1} T^{2}}{24}-\frac{3}{\pi}\left(\frac{2+N_{1}}{3} \frac{\lambda_{1}}{24}\right)^{\frac{3}{2}} T^{2}
$$

At this stage, one would be tempted to improve the result eq. (16), by incorporating also the logarithmic terms in the expansion of $h$. This would give us a correction to $\frac{M^{2}}{T^{2}}$ of order $\lambda_{1}^{2} \ln \lambda_{1}$, but, as contributions of this order arise also from two-loops diagrams, it would be necessary to include them as well for consistency, something that we will not attempt in this paper.

As we said above, we were assuming that $m^{2}$ was positive. In fact, the case in which $m^{2}$ is negative can be treated in a completely analogous way, because one is working in the regime of very high temperatures where one "self consistently" assumes that the symmetry is restored. By self consistency we mean that one is able to find solutions to the gap equations of the unbroken phase giving a positive result for $M^{2}$. In this respect, notice that the next to leading correction, although coming with the opposite sign than the leading one (see eq.(16), will not change the sign of the self-energy unless $\lambda_{1}$ becomes large, in which case any attempt of a perturbative calculation becomes meaningless. Thus, in this case the subleading correction cannot alter the symmetry breaking pattern at high $T$.

We now turn to the $O\left(N_{1}\right) \times O\left(N_{2}\right)$ model. There will be a set of two coupled gap equations,

$$
\begin{aligned}
& x_{1}^{2}=\frac{m_{1}^{2}}{T^{2}}+\lambda_{1} \frac{2+N_{1}}{3} h\left(x_{1}\right)+\lambda N_{2} h\left(x_{2}\right), \\
& x_{2}^{2}=\frac{m_{2}^{2}}{T^{2}}+\lambda_{2} \frac{2+N_{2}}{3} h\left(x_{2}\right)+\lambda N_{1} h\left(x_{1}\right),
\end{aligned}
$$

where

$$
x_{i}=M_{i} / T
$$

Using the asymptotic expansion eq.(9), and keeping up to the linear terms, eqs.(17-18) become, for $\frac{T}{m} \gg 1$

$$
\begin{aligned}
& x_{1}^{2}=c_{1}-\lambda_{1} \frac{2+N_{1}}{24 \pi} x_{1}-\lambda \frac{N_{2}}{8 \pi} x_{2}, \\
& x_{2}^{2}=c_{2}-\lambda_{2} \frac{2+N_{2}}{24 \pi} x_{2}-\lambda \frac{N_{1}}{8 \pi} x_{1},
\end{aligned}
$$

where we have introduced the constants $c_{i}$ as

$$
c_{1}=\lambda_{1} \frac{2+N_{1}}{72}+\lambda \frac{N_{2}}{24}
$$




$$
c_{2}=\lambda_{2} \frac{2+N_{2}}{72}+\lambda \frac{N_{1}}{24}
$$

The constants $c_{i}$ give the thermal masses $M_{i}^{2}$ to lowest order:

$$
M_{i}^{2}=c_{i} T^{2}
$$

As noticed in ref. [2], it is possible to take $\lambda$ negative and still satisfying the constraint eq.(3), in such a way that one of these combinations of coupling constants, say $c_{2}$, is negative (notice that due to the constraint eq.(3) $c_{1}$ is necessarily positive), namely:

$$
\begin{gathered}
\lambda<0, \\
\lambda_{1} \lambda_{2}>9 \lambda^{2}, \\
|\lambda|>\frac{2+N_{2}}{3 N_{1}} \lambda_{2} .
\end{gathered}
$$

In this case, to lowest order, eqs.(17) have no self-consistent real solutions and the corresponding region of parameter space was identified by Weinberg as the region of symmetry non-restoration (or rather of symmetry breaking at high $T$, if the symmetry was unbroken at $T=0$ ). The question that now arises is whether the subleading corrections represented by the linear terms in eqs.(20-21) can alter this picture. Notice that, as opposed to the simpler $O(N)$ case, we now have more than one coupling constants and thus next to leading order effects could become dominant even when all the couplings are of the same order of magnitude. Now, eqs. 20-21) represent a pair of parabolae in the plane $x_{1}$ and $x_{2}$ which intersect in the upper right plane (then giving an acceptable solution to the gap equations and signalling symmetry restoration at high $T$ ) if and only if:

$$
8 \pi \frac{c_{2}}{\lambda N_{1}} \leq\left\{-\lambda_{1} \frac{2+N_{1}}{48 \pi}+\left[\left(\lambda_{1} \frac{2+N_{1}}{48 \pi}\right)^{2}+c_{1}\right]^{\frac{1}{2}}\right\}
$$

The corresponding region of symmetry non-restoration is then described by the set of inequalities:

$$
\begin{gathered}
\lambda<0, \\
\lambda_{1} \lambda_{2}>9 \lambda^{2},
\end{gathered}
$$




$$
|\lambda|>\lambda_{2} \frac{2+N_{2}}{3 N_{1}}+|\lambda| \frac{3}{\pi}\left\{-\lambda_{1} \frac{2+N_{1}}{48 \pi}+\left[\left(\lambda_{1} \frac{2+N_{1}}{48 \pi}\right)^{2}+c_{1}\right]^{\frac{1}{2}}\right\} .
$$

By comparing with eqs.(25), it is clear that the inclusion of next-to-leading order corrections reduces the region of the parameter space in which symmetry non-restoration takes place. We observe that even if there is always a region of symmetry non restoration, its size becomes smaller and smaller as $N_{1}$ and $N_{2}$ grow large.

We can think of our model as representing the Higgs sector of a $S U(5)$ grand unified model, like the one considered in ref.[6]. In this case, taking $N_{1}=90, \phi_{1}$ represents the real components of the Higgs in the $\mathbf{4 5}$ representation of the group $S U(5)$, while taking $N_{2}=24, \phi_{2}$ corresponds to its adjoint 24 representation.

A qualitative indication of the importance of the subleading terms can be obtained by plotting the regions (2527), 29-31) at fixed values of one of the coupling constants and of $N_{1}$ and $N_{2}$. Figures (1) and (2) display these regions for $\lambda_{1}=4 / 5$ and $\lambda_{2}=1$ respectively and $N_{1}=90, N_{2}=24$, which correspond to typycal values of the $S U(5)$ parameters considered in ref.[6]. It is clear from both figures that the subleading terms have a non negligible effect, as they reduce (in the considered region of parametrs) by a factor of roughly two the size of the region of symmetry non-restoration.

In conclusion, we have shown that the inclusion of next-to-leading order contributions can modify in a substantial way the symmetry breaking pattern of an $O\left(N_{1}\right) \times O\left(N_{2}\right)$ model at large $T$. Naturally, the phenomenologically interesting models are those in which part of this symmetry is gauged. Consequently, our analysis of the gap equations should be generalized to include this case. The results presented here lead us to expect that subleading corrections will play an important role also in grand-unified models. We expect to report on this issue in a forthcoming publication.

\section{Acknowledgements}

We would like to thank G.Senjanović and Alejandra Melfo for useful discussions and for communicating us their results prior to publication of their work. 


\section{References}

[1] D.A. Kirzhnits and A.D. Linde, Phys. Lett. 42 B, 471 (1972).

[2] S. Weinberg, Phys. Rev. D9, 3357 (1974).

[3] L. Dolan and R. Jachiw, Phys. Rev. 9D, 3320 (1974).

[4] R.N. Mohapatra and G. Senjanović, Phys. Lett. 89 B, 57 (1979); Phys. Rev. Lett. 42, 1651 (1979); Phys. Rev. D20, 3390 (1979).

[5] P. Langacker and S.-Y. Pi, Phys. Rev. Lett. 45, 1 (1980).

[6] G. Dvali, A. Melfo and G. Senjanović, "Is there a Monopole Problem ?", IC/95/145, SISSA Ref.81/95/A, hep-ph/9507230.

[7] N. Banerjee and S. Mallik, Phys. Rev. D43, 3368 (1991);

R.R. Parwani, Phys. Rev. D45, 4695 (1992). 


\section{FIGURE CAPTIONS}

Figure 1. [Region of symmetry non-restoration at fixed $\lambda_{1}=4 / 5$ for $N_{1}=90$ and $N_{2}=24$. The zeroth order region is the one enclosed by the solid curves, while the region obtained by including next-to-leading order corrections is the one enclosed by the upper solid line and the dashed line.]

Figure 2. [Region of symmetry non-restoration at fixed $\lambda_{2}=1$ for $N_{1}=90$ and $N_{2}=24$. The zeroth order region is the one enclosed by the solid curves, while the region obtained by including next-to-leading order corrections is the one enclosed by the upper solid line and the dashed line.] 

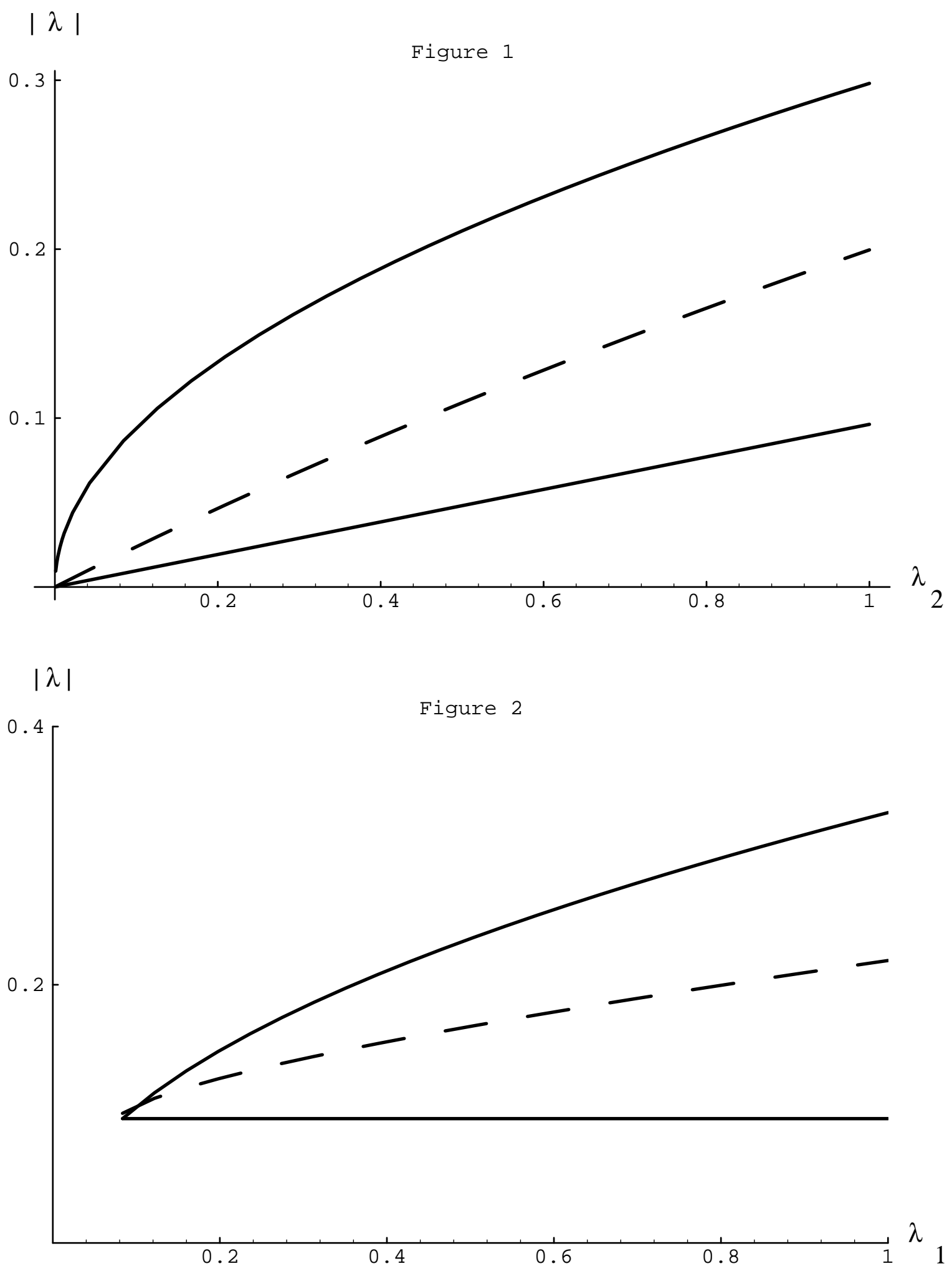